\title{
Real and simulated mechanical tests
}

\author{
Mihai Gabriel MATACHE ${ }^{1, a}{ }^{*}$, Petru CARDEI ${ }^{2, b}$, Gheorghe VOICU ${ }^{3, c}$, \\ Valentin VLADUT ${ }^{4, d}$, Raluca SFARU ${ }^{5, e}$ and Mihai LUDIG ${ }^{6, f}$ \\ 1,2,4,5,6 National Institute of Research-Development for Machines and Installations Designed to \\ Agriculture and Food Industry - INMA, Ion lonescu de la Brad, no. 6, Bucharest, Romania \\ ${ }^{3}$ The Faculty of Biotechnical Systems Engineering, Polytechnic University, Bucharest, Romania \\ agabimatache@yahoo.com, betru_cardei@yahoo.com,

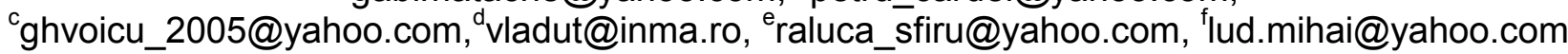

Keywords: physical tests, simulated tests, mechanic

\begin{abstract}
This paper presents the results obtained by the authors when applying mechanical test methods that are validated using fatigue testing under simulated regime with finite element analysis. In addition to the mostly mandatory physical tests, the simulated regime testing can validate emerging conclusions. Virtual testing conclusions (computer simulated via Cosmos finite element analysis software), strengthen, complement and increase the accuracy of physical test conclusions. During the investigation of the physical test results, there are cases where the simulated physical test is not always explainable. This paper presents a case study conducted on special constructed screws that are subjected to fatigue and verified using the finite element analysis. It presents the possibility for practical application of simulated tests in order to redesign structures that fail during physical tests.
\end{abstract}

\section{Introduction}

The paper comments and highlights the critical laboratory tests aspects which should give extremely important conclusions for high safety elements.

Fatigue testing of structures with high safety requirements needs the load propping structure to be as close to reality as possible [1,2,3]. Otherwise, the results will underestimate or overestimate (in the worst case) the tested structure. The propping and loading (stress) structure is a sensitive issue and the realization of more physical variants of the test stand in order to choose the best one between them in terms of closeness to reality, is expensive. Also, structures of this type are expensive, so the destruction of a large number of such structures is itself costly. In order to reduce test cost, the authors want to describe two ways of solving the problem. The first is the very close collaboration with the stand producer when designing the test structure. The second is to run a real test in parallel with one or more virtual tests using one or more simulators based on various mathematical models.

Collaborating with the structure producer in the design stage of the testing stand is mandatory at least for structures with high degree of safety. The introduction of equivalent bonds (stiffeners, jams, and so on) and the application of the loads are often simulation elements (does not apply just like in reality because the tested item do not work on the stand in the assembly in which it operates in reality) which are working hypotheses belonging to the recipient and tester's responsibility. For these reasons, the construction of the prop and loading on the testing structure, both in terms of physical design and realization belong to the recipient and the person that is testing.

\section{Materials and experimental procedure}

Within the paper there are presented experimental results obtained during static and dynamic resistance tests of some special type screws in controlled conditions, followed by virtually simulated tests. 
To begin with, some general characteristics of the screw (bolt) are shown which form the subject of these tests. The screw is a relatively simple metallic cylindrical shape element with a diameter varying between 19 and $22 \mathrm{~mm}$ and a reference length of $80 \mathrm{~mm}$. At one end is fitted with a M221.5 thread and on the other end with a truncated locking head. The screw is made of steel $16 \mathrm{NiCr} 4$.

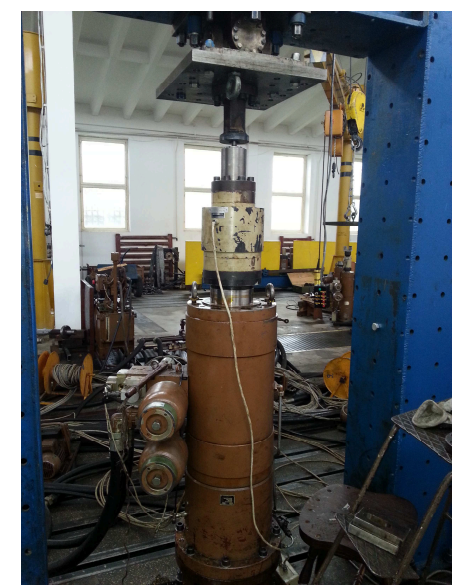

Fig. 1 Physical testing installation

The $16 \mathrm{NiCr} 4$ steel has many features given in common material data from web pages [4]. Important characteristics in terms of these tests are as follows: ultimate tensile strenght, 900.83 $\mathrm{MPa}$, yield tensile strength, $895.59 \mathrm{MPa}$, and also the break elongation 12\%. According to [5] the fatigue tension limit for the steel should be between 315 and $450 \mathrm{MPa}$. If you consider Table 14.2, page 315, at [6], then, for pure bending, the fatigue endurance limit would be about $360 \mathrm{MPa}$, traction - compression between 540 and $810 \mathrm{MPa}$, and for torsion between 198 and $225 \mathrm{MPa}$. These limits are typical for breaking tension limit above $700 \mathrm{MPa}$.

Physical tests to which the screw was subjected were:

-monotone linear force load test up to breakage

-dynamic fatigue test using a $6 \mathrm{~Hz}$ frequency sinusoidal signal.

Both types of tests were performed on a mini-stand that was built inside the testing laboratory. We used a $400 \mathrm{kN}$ hydraulic cylinder mounted under a $U$ frame. We have attached to the frame a three degree of freedom joint fitted with a rig designed to hold the screw. On top of the cylinder we mounted another rig to hold the nut, and between the two rigs we have put the screw.

Testing under simulated regime means virtual tests, having a standard or nonstandard mathematical base model. Besides virtual tests we used small-scale tests or analog tests. The standard mathematical models used in structural virtual tests for elements that are tested in the laboratory or on the field, are the classical models of continuous body from scientific literature of elasticity, plasticity, strength of materials, and so on [7]. Nonstandard models are mathematical models of a new type, still not available in the scientific literature. These models try to simulate the accumulation of fatigue during functioning of some structural elements for example.

The general problem of propping and testing structures under normal or simulated regime is very delicate. In fact constraining and loading of a structure means the same phenomenon for the structure, namely its contact with the external environment, which takes place in very different ways. Reaction forces exist in constrains, which are calculated using mathematical models. For this reason, some programs already named the structural analysis from both categories (propping and loading) with a single word: stress or loads. Obviously loads are given not only in forces but also in initial conditions, displacements, velocities, accelerations, and so on.

Simulated test systems of the screw were performed on a simulator based on a structural model solved using the finite element method. The model is three-dimensional and has the following main components: geometry, support and loading, namely the type of structure material (steel $16 \mathrm{Ni} \mathrm{Cr} 4$ ). 
Structural model of the screw. The screw geometry is very close to reality. A graphical representation of the screw geometry is given in Fig. 2, in medallion appearing the physical model. The only real deviation from the model is that the thread is circular and not spiral. However, the model achieves its goals of studying the phenomena that occur based on the thread flanks and due to their small tilt.

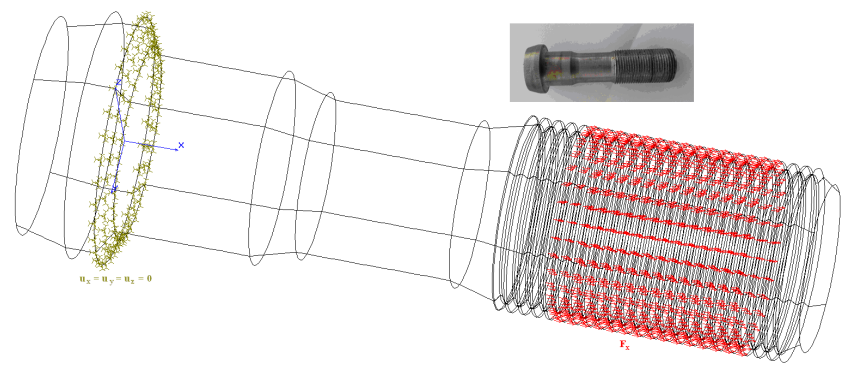

Fig. 2 Structural model geometry of the screw

Constrains on the model were made by canceling linear movement and in all three directions in each node on the circular crown at the base of the screw head. Loading is on the flanks of the thread in the positive direction of the $\mathrm{x}$-axis in 1440 nodes each with $186181 \mathrm{~N}$.

In all calculation must be kept in mind that the length of the bolt stretch (the length between the bearing and the center of the first turn of the thread fastened in the nut which transmits the force). This length is calculated as the distance between the centers of the corresponding cross sections of the screw.

\section{Results and discussions}

Quasi-static testing for tension is performed under constant loading speed by applying a linearly increasing force to the point when the screw breaks. Maximum traction force value was $268.1 \mathrm{kN}$. The range of the elasticity curve is shown in Fig. 3 The maximum specific deformation from the linear-elastic range is around 0.001 , with $210 \mathrm{MPa}$ pressure and the force at which it is reached of about $59540 \mathrm{~N}$. The maximum relative displacement on the corresponding traction axis is approximate $0.048 \mathrm{~mm}$. Due to the resolution and high relative displacement ratio of the elastic landing, it is practically invisible on the graphical representation.

A picture of a screw broken during physical pure tension testing is shown in Fig. 4. Note that the failure occurred in the minimum diameter area of the screw $(19 \mathrm{~mm})$. No significant changes were observed on the thread walls.

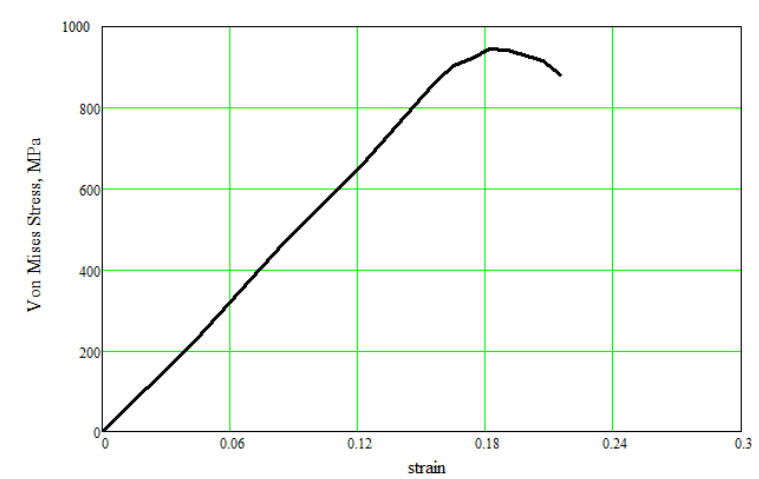

Fig. 3 Tension curve - specific deformation

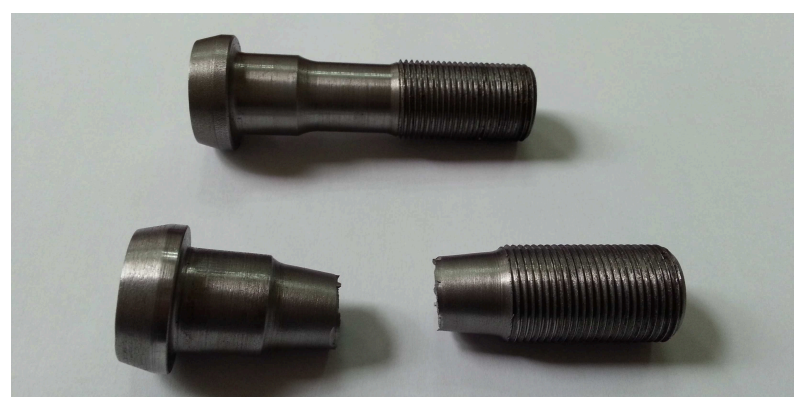

Fig. 4 Screw after the quasi-static traction test

Dynamic fatigue testing was materialized by subjecting the screw to sinusoidal stress of constant frequency and amplitude. Therefore the test frequency was $6 \mathrm{~Hz}$ with a signal amplitude of $72900 \mathrm{~N}$ around the mean value of $89100 \mathrm{~N}$. The cycles were counted for each screw up to breaking. In Fig. 5 a sequence of force recording is shown with which the screws were stressed. In fig. 6 , we see the section in which the screw broke as it was at the lower screwing limit in the nut 
that drives the traction force. Also the appearance in the cross section where the screw failure occurs is classic for breaking due to fatigue accumulation, [8].

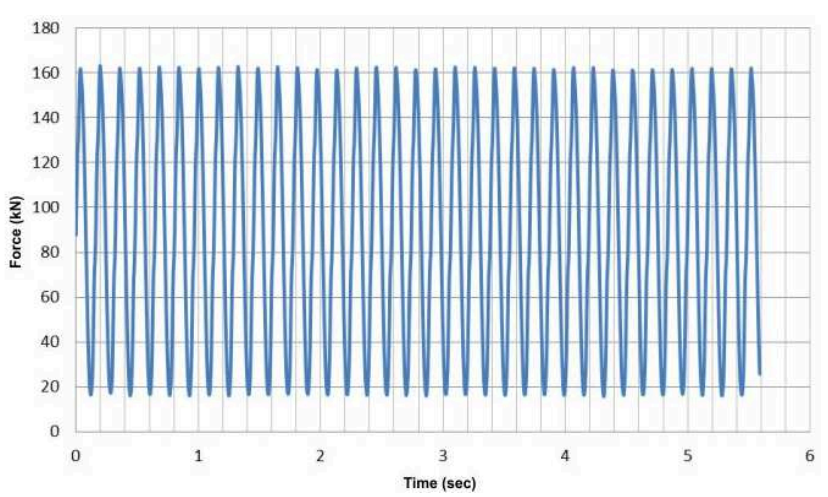

Fig. 5 Stress force cyclic sequence

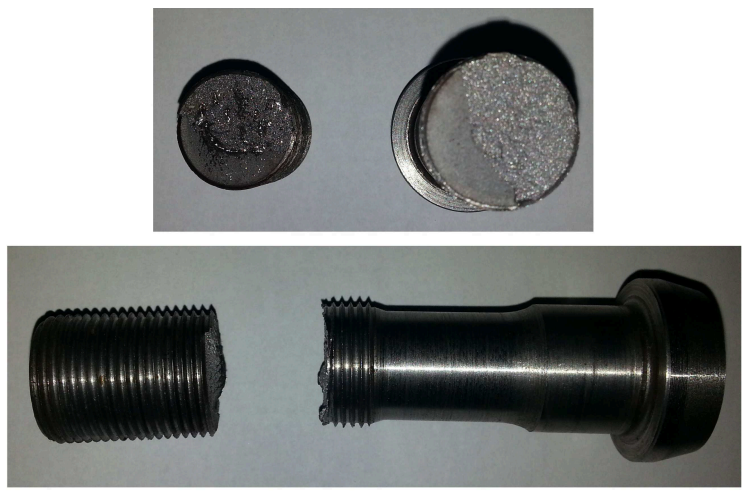

Fig. 6. Special screw broken during cyclic tests

Static stress test result under simulated regime. In case of the physical stress test by stretching up to breaking, the load model is that from Fig. 3, indicating that the force increases from 0 to the value of $186.181 \mathrm{~N}$ in each of the 1440 nodes in which it is applied, therefore a total of $268100 \mathrm{~N}$. This load is perfect, assuming that the contact between the screw and the nut threads is ideal. Consequently all the turns from the length of the contact between the screw and the nut are evenly loaded. Traction occurs in the Ox direction, also being ideal, no lateral deviations. For accurate simulation of the stretching test, up to breaking, it is mandatory that the material follows the characteristic curve for stretching, so that it will include linear elastic range (very small in size compared to the relative displacement of the screw from the rest of the test), a possible nonlinear range, elastic and plastic range, which includes plastic irreversible deformation.

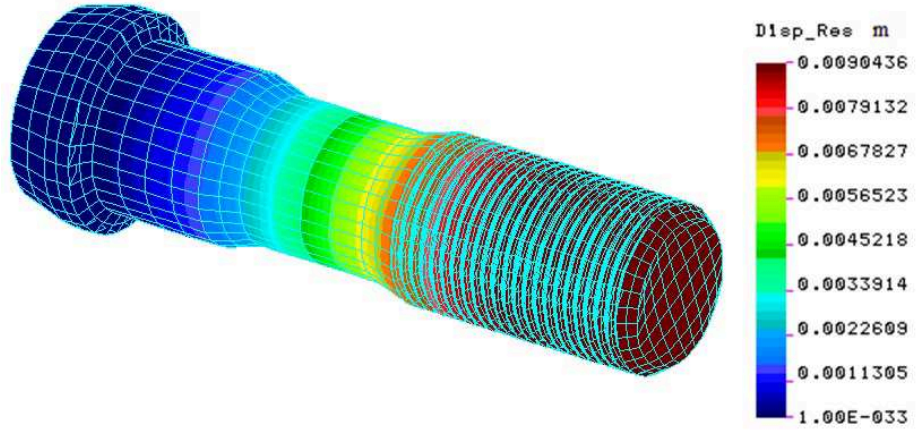

Fig. 7 Resultant relative displacement map (deformation) bolt structural model boundary at time $\mathrm{t}=$ $1 \mathrm{~s}$ (the last step of loading, 38)

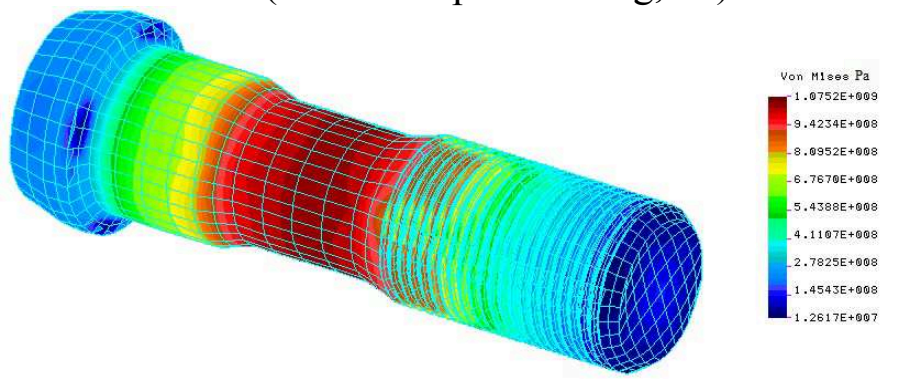

Fig. 8 Equivalent stress map (Von Mises, [9]) bolt structural model boundary at time $\mathrm{t}=1 \mathrm{~s}$ (the last step of loading, 38) 
As seen in Fig. 7 the relative displacement resulted maximum in the structural model of the straight stretched bolt up to breakage, has on the boundary, a maximum value of $9.04 \mathrm{~mm}$. This value is localized in an area near the bolt grip jaws from stretching force. It is reminded that this is improperly called static test because the load varies over time, not instantly reaching the maximum value that breaks the structure. Also remember that the nut jaws acting with tensile force, include almost the entire screw thread. Note that a high value of relative displacement occurs at the first turns of the thread which are fastened on the laws from the central area of the screw.

An axial section equivalent von Misses stress in screw, in case of "static" stress, is given in Fig. 8. It can be observed that the extreme values are localized in the center of the bolt toward the outer border. This explains why failure occurs on the center of the bolt, as confirmed by the physical experiment seen in fig. 4 .

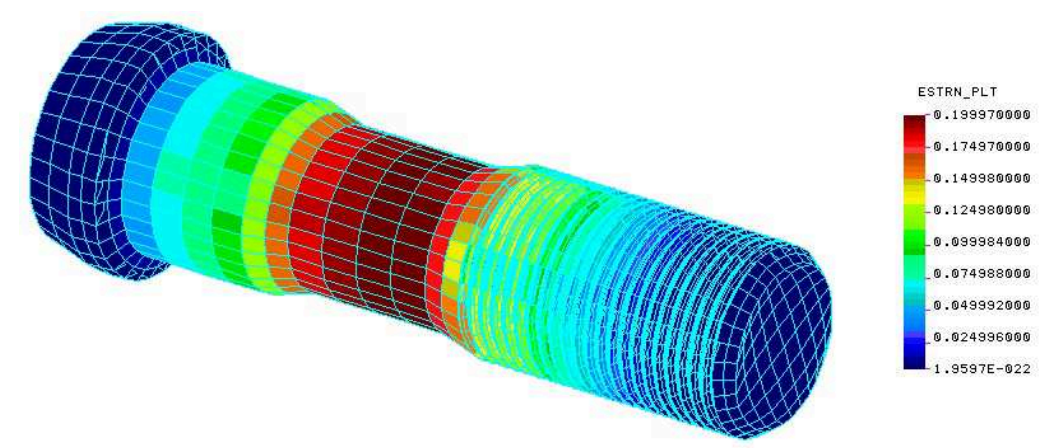

Fig. 9 Plastic specific deformation map on the boundary of the structural model of the bolt at the final time, $\mathrm{t}=1 \mathrm{~s}$ and integration step 38

Although called "static" this quasi-static simulation (low speed linear load force) allows the timemovement tracking of all the points from the structure. From these, we extracted few of the most exciting, located around the area of high stress.

The response in the plastic structure is also characterized by the specific deformation maps. Note that the maximum values are clearly on the central part of the bolt, where the test structure eventually breaks. The specific equivalent strain distribution map or total plastic deformation (or plastic deformation share of the total) is given in Fig. 9. The map clearly shows the structural model of the screw at the last integration step, step 38, and final load time, $1 \mathrm{~s}$, when failure occurs, within this time limit.

We emphasize again that in this numerical experiment, the "static" test is modeled in a manner much closer to reality. The structural model is a dynamical type and loads the screw in one second to the maximum value at which rupture occurs. Loading speed is small compared with the speed of dynamic cyclic stress test so that this stretch test can be called quasi-static breaking test. The analysis is developed in 38 integration steps with variable time length.

The simulator results, in terms of quasi-static load test before break are consistent with physical test results - screw linear stretching experiment up to breaking.

Results of the simulation periodical stress test. This test is usually performed in order to establish the resistance to fatigue. Load and support are in the same location and with the same intensity as quasi-static testing. The minimum value of force is $16200 \mathrm{~N}$ and the maximum is $162000 \mathrm{~N}$.

The structural model simulates the cyclic stress of the bolt through a load variable in time, with a frequency of $6 \mathrm{~Hz}$, as in reality. According to the procedure from the structural analysis software used (COSMOS 2.8), the force with which the model is loaded, is constant and equal to the maximum cyclic force and the variation is given by multiplying it by a coefficient that varies the same as the real force, but with values scaled by dividing the maximum value of the force. The maximum loading force was $162000 \mathrm{~N}$, and the minimum was in cycles of $16200 \mathrm{~N}$. 
The variation coefficient for the maximum amplification force for applying the cyclic force on the screw is plotted in Fig. 10. Notice that we introduced in the simulation, the transitory phase of linear load from value of 0 force to the mean value after which we followed a sinusoidal signal with an mean values of 0.55 , a minimum of 0.1 and a maximum of 1 . This coefficient multiplies the $162000 \mathrm{~N}$ force applied on the thread flanks of each node in 1440 nodes each with $112.5 \mathrm{~N}$ thereby simulating the force applied on the real model. We introduced the transitional limit of discontinuities and influences to make the simulation as close to reality as possible.

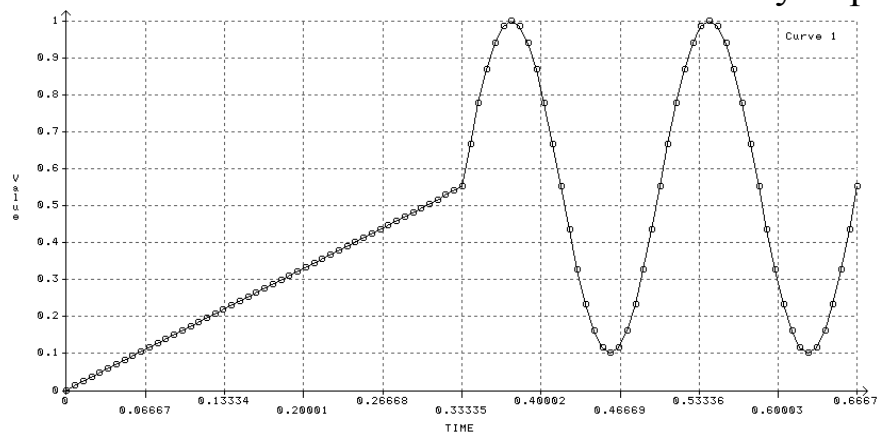

Fig. 10 Variation of the maximum force amplification coefficient in time

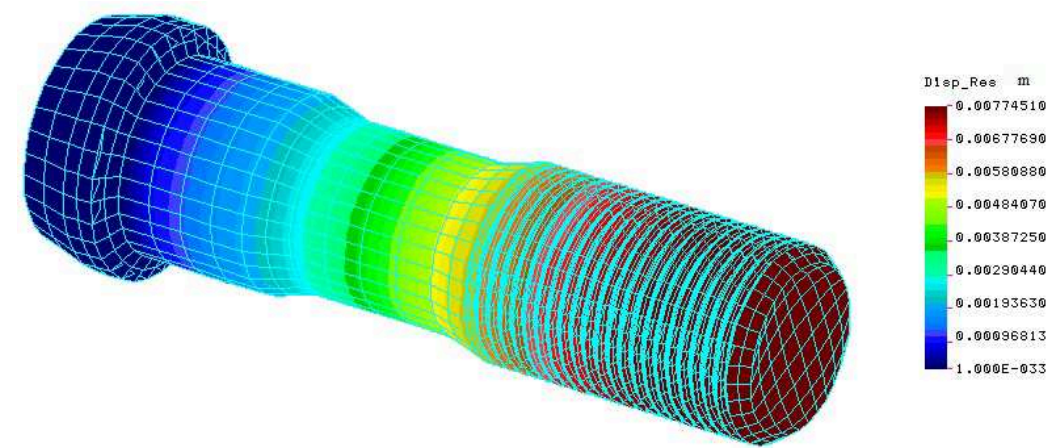

Fig. 11 The resulted relative displacement field map on the boundary of the structural model of the screw at step 86 , and time $\mathrm{t}=0.36893 \mathrm{~s}$

Color map of relative displacement field map on the boundary of the structural model of the screw is given in Fig. 11, for the first peak load at $t=0.36893$ s. Note that the maximum displacement, where the force is greater (as in the case of quasi-static tensile test, fig. 7), while the minimum is at the support area. The maximum relative displacement resulting from this peak load is $7.74 \mathrm{~mm}$. It is obvious that the screw enters the field of nonlinear elastic and plastic working regime.

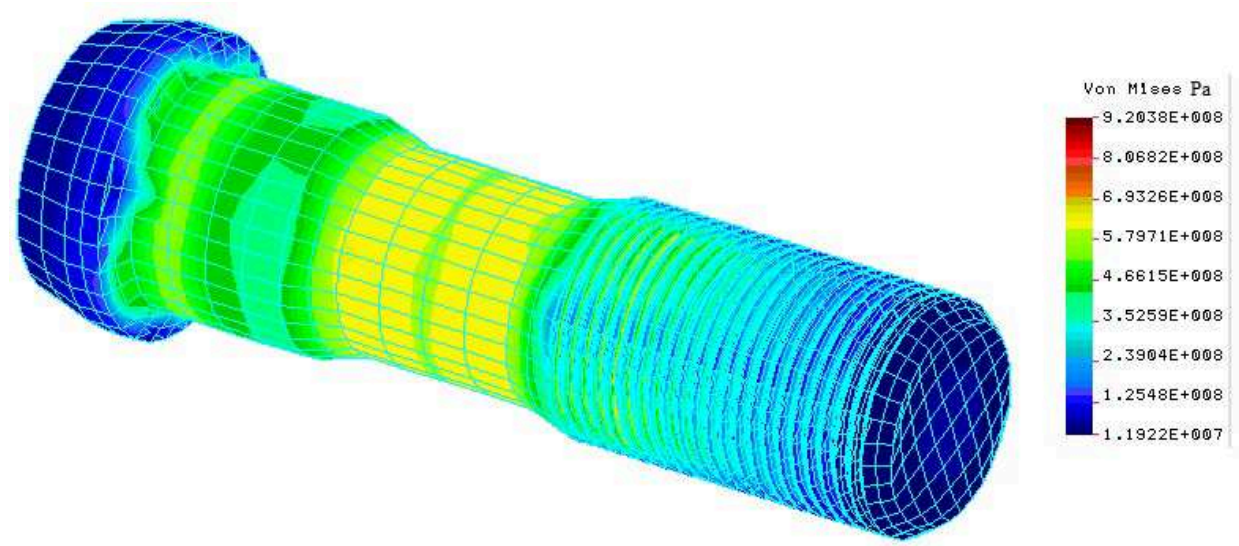

Fig. 12 Equivalent tension field map, (plastical), on the screw structural model boundary at step 86, and time $\mathrm{t}=0.36893 \mathrm{~s}$ 
As it can be seen from the loading in the non-linear elastic-plastic model linear the maximum equivalent structural model boundary stress is $920 \mathrm{MPa}$ (Fig. 12), this being the tension value located near the breakage point of the material. A detail of the threaded area (fig. 13) for the plastic elastic-linear case, shows that the maximum equivalent stress is reached only in very small areas of volume, based on the first two turns of the thread flanks fastened by the traction nut, counting from the center of the bolt. The equivalent high tension found at the flanks base and the other are caught in the driving nut. The maximum equivalent stress is distributed in several areas at the base of some flanks, but the largest volume is at the base of the flank of the first turn that is caught in the nut, counting from the bolt's structural model center.

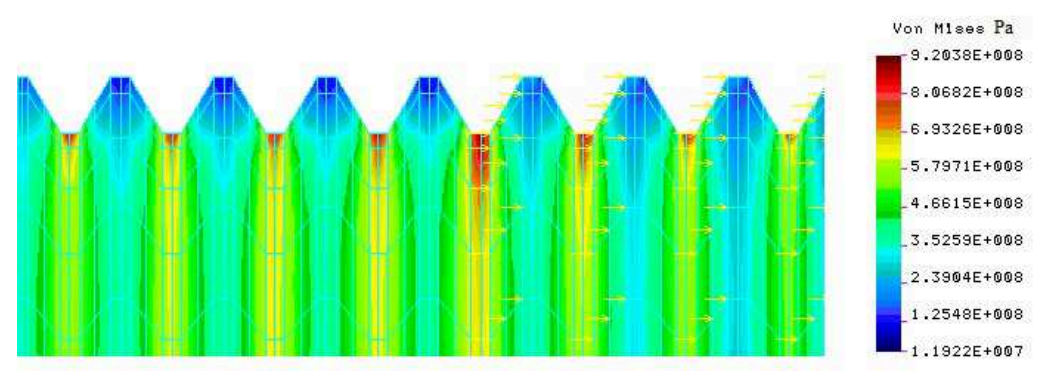

Fig.13 Equivalent boundary map of tension field on the structural model of the screw (detail of the overstretched flanks of the thread), at step 86 , and time $\mathrm{t}=0.36893 \mathrm{~s}$

For nonlinear elastical-plastical analysis, the COSMOS / M 2.8 software was run with the option of variable step using loading time curve from Fig. 10. As in the static analysis of the structural model, it was meshed with 12544 screw SOLID type elements with eight nodes and 15957 nodes.

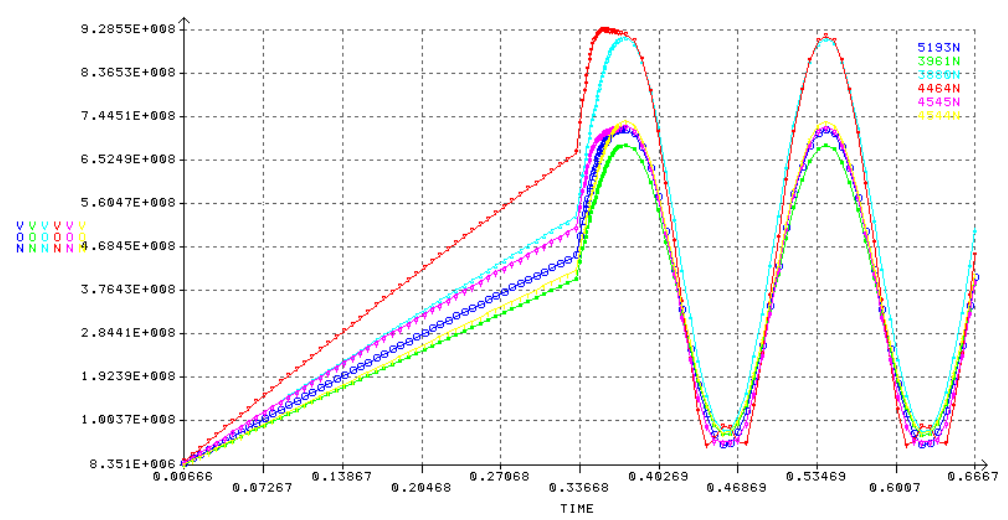

Fig. 14 Time variation of equivalent stress (von Mises) in six nodes from the high stress area of the structural model of the screw (the base between two flanks of the thread - the border between the first and second turn in contact with the drive nut, counted from the center of the screw)

\section{Conclusions}

The main conclusions of the structural analysis of the special purpose screw described in this paper, are the following:

It can be seen that in the elastic linear range of the material, the relative displacements (deformations) are insignificant and linear. Gradually, after entering the elastic and plastic nonlinear range, displacements grow appreciably in relation to the active linear elastic region and, in addition, become nonlinear, more pronounced, until the time of breakage. The simulated test results confirm the physical test results.

Linear elastic static analysis is not relevant for cyclic loading test until failure by fatigue.

Significant minimal test for linear traction test up to breaking and for the cyclic dynamical testing up to breaking due to fatigue is the elastical-plastical nonlinear dynamic analysis. This means one 
has to account for the tension-deformation characteristic curve of the material at pure traction, the loading time curve scaled to maximum force and the support at maximum load. Also the initial time and final time of the test are taken into account.

The structural model of the screw, which is its simulator or its virtual structure, indicates that failure when performing linear loading on the screw up to breaking occurs in the middle crosssection of minimum diameter. Though highly stressed areas at the base of the first turn fastened on the driving nut, these areas do not broken because they have a very small spatial extent, but in relation to the minimum diameter, the central area, where the surface on which the equivalent tension is higher than the tension breaking limit of the material, reaches the cross section area.

The structural model of the screw also indicates that the screw fatigue failure when doing cyclic loading occurs in the area of the first turn of thread fastened in the driving nut. This conclusion is consistent with the suggestion from [10].

\section{References}

[1] Q. H. Vu, D. Halm, Y. Nadot, Multiaxial fatigue criterion for complex loading based on stress invariants, International Journal of Fatigue-INT J. FATIGUE 01/2010; 32(7):1004-1014.

[2] C. Froustey, S. Lasserre, Multiaxial fatigue endurance of 30NCD16 steel, International Journal of Fatigue, vol. 11, issue 3, may 1989, p. 169-175.

[3] L. Susmel, Multiaxial Notch Fatigue, Woodhead Publishing in Materials, 2009.

[4] Information on http://www.steelnumber.com/en/steel_composition_eu.php?name_id=238.

[5] Information on http://www.roymech.co.uk/Useful_Tables/Fatigue/Fatigue.html.

$[6]$ Information on

http://www.google.ro/search?q=axle+fatigue+failures + photo\&newwindow $=1 \&$ tbm $=$ isch $\&$ tbo $=u \& s$ ource=univ\&sa=X\&ei=7_h0U4fFK-WN4gS6uoD4BA\&ved=0CCoQsAQ\&biw=1366\&bih=667.

[7] J, C. Newman, R., S. Piascik, Fatigue Crack Growth Thresholds, Endurance Limits, and Design, ASTM, STP 1372, Issue 1372,2000.

[8] V. Drobota, Materials resistance, Didactic and Pedagogical Publishing House, Bucharest, 1982, p. 309.

[9] Gh. Buzdugan, Materials resistance, Technical Publishing House, Bucharest, 1982.

[10] Gh. Manea, Machineries organs, vol. I, Technical Publishing House, Bucharest, 1970, p. 317. 